\title{
Ceramic-on-Ceramic Total Hip Arthroplasty with Large Diameter Heads: A Systematic Review
}

\author{
Francesco Castagnini $^{a} \quad$ Monica Cosentino ${ }^{b} \quad$ Giovanni Braccia $^{a}$ Claudio Masetti $^{a}$ \\ Cesare Faldini ${ }^{c, d}$ Francesco Traina ${ }^{a, d, e}$ \\ ${ }^{a}$ Ortopedia-Traumatologia e Chirurgia Protesica e dei Reimpianti d'anca e di Ginocchio, IRCCS Istituto Ortopedico

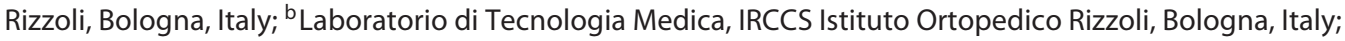

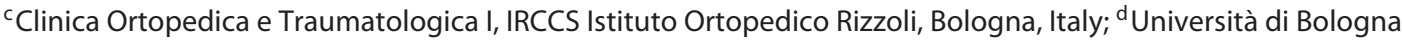

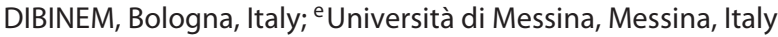

\section{Highlights of the Study}

- Clinical and radiological outcomes of ceramic-on-ceramic total hip arthroplasty with large-diameter heads were excellent in the short- to mid-term, with minimal revision rates

- Dislocations occurred in $<1 \%$ of the cases

- Failures of the bearing surfaces were not observed

- Noise was reported in up to $30 \%$ of the cases

- Noise was inconstantly associated with implant positioning, younger age, and larger heads.

\section{Keywords}

Delta bearing - Total hip arthroplasty · Ceramic-on-ceramic coupling

\begin{abstract}
Ceramic-on-ceramic (COC) total hip arthroplasties (THAs) with large heads (>36 $\mathrm{mm}$ ) were introduced to reduce dislocation rates and restore the hip anatomy as closely as possible to the native one. To date, the literature is scarce and fragmented; a review is desirable to point out the outcomes and the possible specific complications (noise, groin pain, and taperosis). A systematic review about large-diameter COC THAs was conducted according to the PRISMA guidelines. The PubMed and Cochrane databases were searched
\end{abstract}

karger@karger.com www.karger.com/mpp

Karger $\stackrel{\text { ' }}{5}$

GOPEN ACCESS
(C) 2020 The Author(s)

Published by S. Karger AG, Basel

This is an Open Access article licensed under the Creative Commons Attribution-NonCommercial-4.0 International License (CC BY-NC) (http://www.karger.com/Services/OpenAccessLicense), applicable to the online version of the article only. Usage and distribution for commercial purposes requires written permission. using the terms "large", "big", "head", "hip", and "ceramic." The methodological quality of the papers was assessed using the MINORS (Methodological Index for Nonrandomized Studies) score. Seven papers (level of evidence: 5 case series and 2 case-control studies) met the inclusion criteria. Clinical outcomes were excellent in $>90 \%$ of the patients. Groin pain was reported in only 1 article (7\%). Radiological outcomes were positive. Minimal revision rates $(<2 \%)$ were reported at short- to mid-term follow-ups. Dislocation rates were usually $<1 \%$. No bearing surfaces failed. No case of trunnionitis was reported. Noise occurred in up to $30 \%$ of the patients, mostly squeaking. The noise did not influence clinical outcomes or patients' satisfaction. Noise was inconstantly associated with component positioning, younger age, high articular excursions, and larger sizes. Large-diameter heads in COC THAs 
$(\geq 40 \mathrm{~mm}$ ) showed promising clinical and radiological outcomes with minimal revision rates. Noise is a cause of concern and should be carefully evaluated at longer follow-ups and in larger, prospective, and specifically designed case series.

(c) 2020 The Author(s)

Published by S. Karger AG, Basel

\section{Introduction}

There is a growing interest in large-head-diameter, ceramic-on-ceramic (COC) total hip arthroplasty (THA) [1-4]. This implant should theoretically overcome the drawbacks of the large metal-on-metal implant (adverse local tissue reactions), while maintaining the improved stability and the closer restoration of the hip anatomy provided by the large head $[1,2]$. However, many concerns are related to the use of large-head-diameter COC THAs, like groin pain, noise, bearing fractures, aseptic loosening, and trunnionitis [1-4].

The 2019 Australian registry report highlighted that, in COC couplings, $\geq 40$-mm heads were less prone to dislocation than all smaller-head diameters $(0.2 \%$ of revisions due to dislocations) [5]. While this finding supports the use of large-head COC THAs, the instances of very high friction, documented by Bishop et al. [6] in adverse conditions of lubrication, are not reassuring, and are potential noteworthy drawbacks of these implants.

Thus, a systematic review on large-head-diameter $(\geq 40 \mathrm{~mm}$ ) COC THAs was conducted, to evaluate the clinical outcomes and the potential complications of these implants, paying special attention to: dislocation, noise, component fracture, aseptic loosening, and trunnionitis.

\section{Methods}

A systematic review on COC THAs with a large head size was conducted according to the PRISMA guidelines [7]. The PubMed and Cochrane databases were searched (May 3, 2020). The search was performed using the terms "large", "big", "head", "hip", and "ceramic". In PubMed, the search string was: (((large) OR big) AND hip) AND head) AND ceramic. In the Cochrane database, it was: (( (large) OR big) AND hip) AND head) AND ceramic in (in the title, abstract, and keywords). The articles were screened by 2 independent reviewers: the first step included a selection by title and abstract. The inclusion criteria for relevant articles were: all levels of evidence, English language studies, and no time limitation. Exclusion criteria were: reviews, expert opinions, book chapters, case reports, in vitro studies, and non-English language studies. In the second step, the full texts of the relevant studies were evaluated; articles including $<80 \%$ of COC THAs with $\geq 40-\mathrm{mm}$ heads were excluded. The reference lists of the selected papers were screened for potential inclusive studies (Fig. 1).

The methodological quality of the included papers was assessed using the MINORS (Methodological Index for Nonrandomized Studies) score [8]. The investigations of clinical and radiological outcomes (with a special focus on noise), revision rates, and reasons for revision made the studies adequate. A minimum followup of 5 years was considered adequate, as ceramic-related complications tend to occur by 5 years after implantation [9]. Relevant data about demographics, implant-related features, clinical outcomes, radiological outcomes, and complications were recorded.

\section{Results}

Only 7 papers met the inclusion criteria (Table 1) [1016]. Five were non-comparative studies and 2 were comparative studies. Six studies were prospectively designed. The main features of the papers are summarized in Table 1: all papers were published between 2013 and 2020 [10-16]. All papers reporting non-comparative studies achieved a high-quality MINORS score $(>12$ points). Of the comparative studies, 1 paper met the high-quality threshold ( 20 points). The mean MINORS score was 13.6 points for non-comparative studies and 19.5 points for comparative studies.

\section{Demographics and Implant-Related Features}

The 5 non-comparative studies encompassed >100 hips; the largest case series was described by Blakeney et al. [12] and 264 implants were assessed $[10,11,14,15]$ (Tables 1, 2). The study by Goldhofer et al. [14] was an update of Tai et al. [11]. The comparative studies involved 27 and $57 \mathrm{im}$ plants, respectively; the largest case-control study by Eichler et al. [16] was an update of Deny et al. [13].

All 7 studies included quite a young population (mean age $<70$ years) and a female prevalence (Table 2) [10-16]. More than $80 \%$ of the THAs were implanted due to osteoarthritis. McDonnell et al. [10] specifically selected a population of patients at a high risk of instability. Two types of implants were adopted, the Maxera LDH (Zimmer, Warsaw, IN, USA) and the DeltaMotion Hip System (DePuy Synthes, Warsaw, IN, USA) (Table 1) [10-16]. The minimum rate of large heads $(\geq 40 \mathrm{~mm})$ was $88 \%$ [15]. All the papers reported short- to mid-term followups ranging from 21 to 78.8 months (Table 2) [10-16]. All the implants achieved a minimum follow-up of 1 year.

\section{Clinical Outcomes}

Tai et al. [11], Goldhofer et al. [14], and Lavigne et al. [15] provided a clinical assessment using the HHS (Harris
Med Princ Pract 2021;30:29-36 DOI: $10.1159 / 000508982$
Castagnini/Cosentino/Bracci/Masetti/ Faldini/Traina 


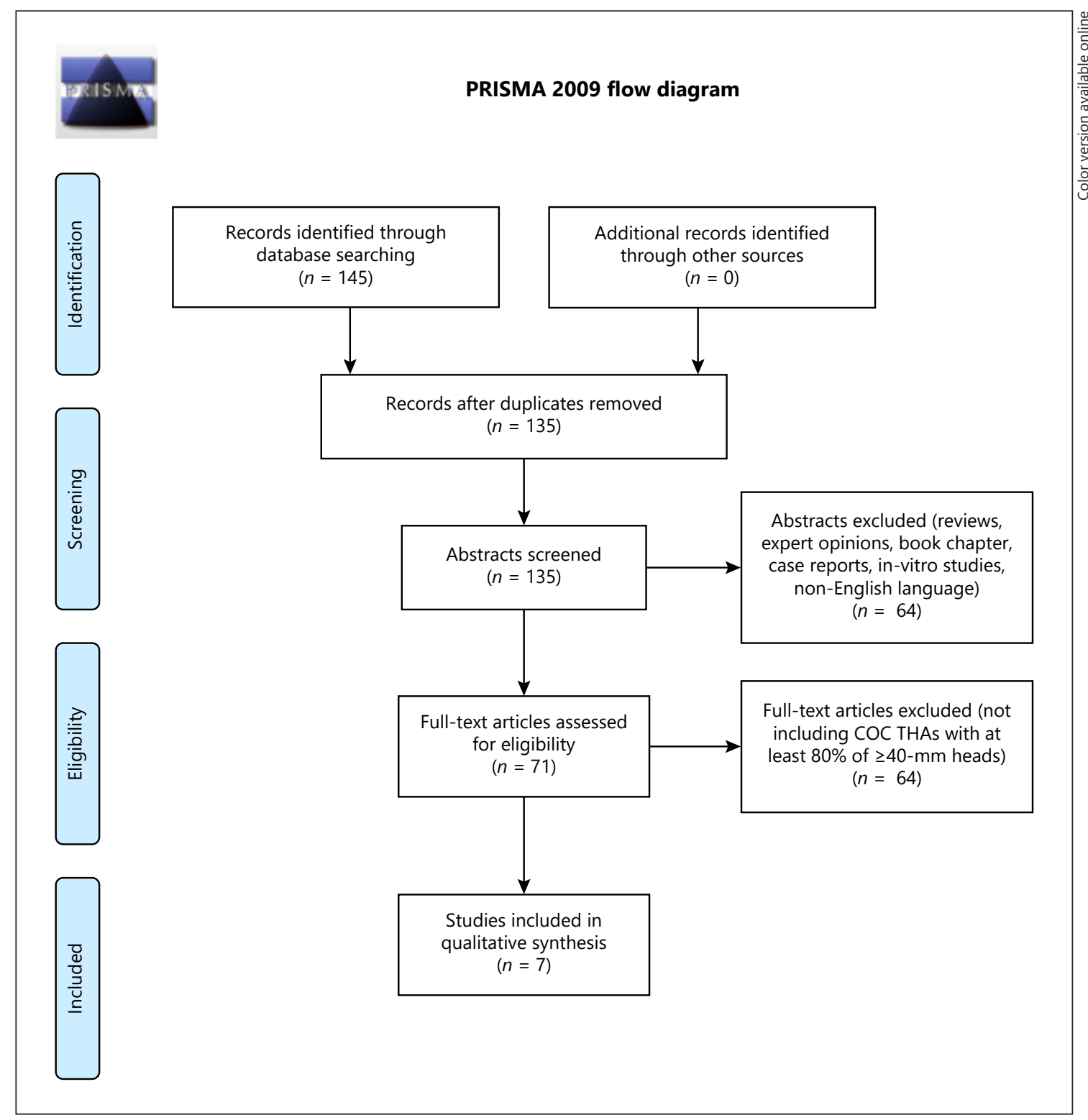

Fig. 1. PRISMA flowchart showing that only 7 articles met the inclusion criteria.

Hip Score), reporting a mean excellent outcome at the final follow-up (Table 3). Blakeney et al. [12] described very positive outcomes, providing other assessments: a UCLA (University of California, LA) Activity Scale score of 6.6, an SF-12 (12-Item Short-Form Health Survey) MCS (Mental Component Summary) score of 52.8 and PCS (Physical Component Summary) score of 53.0, a WOMAC (Western Ontario and McMaster Universities Osteoarthritis Index) score of 7.7, and a FJS (Forgotten Joint Score) of 88.5 (Table 3). Excellent outcomes and patient satisfaction were reported in $>90 \%$ of cases $[11,12$,
14]. Lavigne et al. [15] noticed a lower rate of painless hips $(81.8 \%), 98.8 \%$ of their patients were satisfied, and $63 \%$ were very active. The "sensation of natural hip" was investigated by Blakeney et al. [12] and Eichler et al. [16] and was reported by $74 \%$ and $40 \%$ of patients, respectively.

McDonnell et al. [10] reported the clinical outcomes of large-head THAs after stratification into "silent" and "squeaking" hips (Table 3). The HOOS (Hip Osteoarthritis Outcome Score), VR-12 (Veterans RAND 12-Item Health Survey), Oxford Hip Score, and UCLA Activity 
Table 1. The papers were rated according to the MINORS score: a minimum follow-up of 5 years was considered adequate, as many ceramic-related complications tend to occur by 5 years

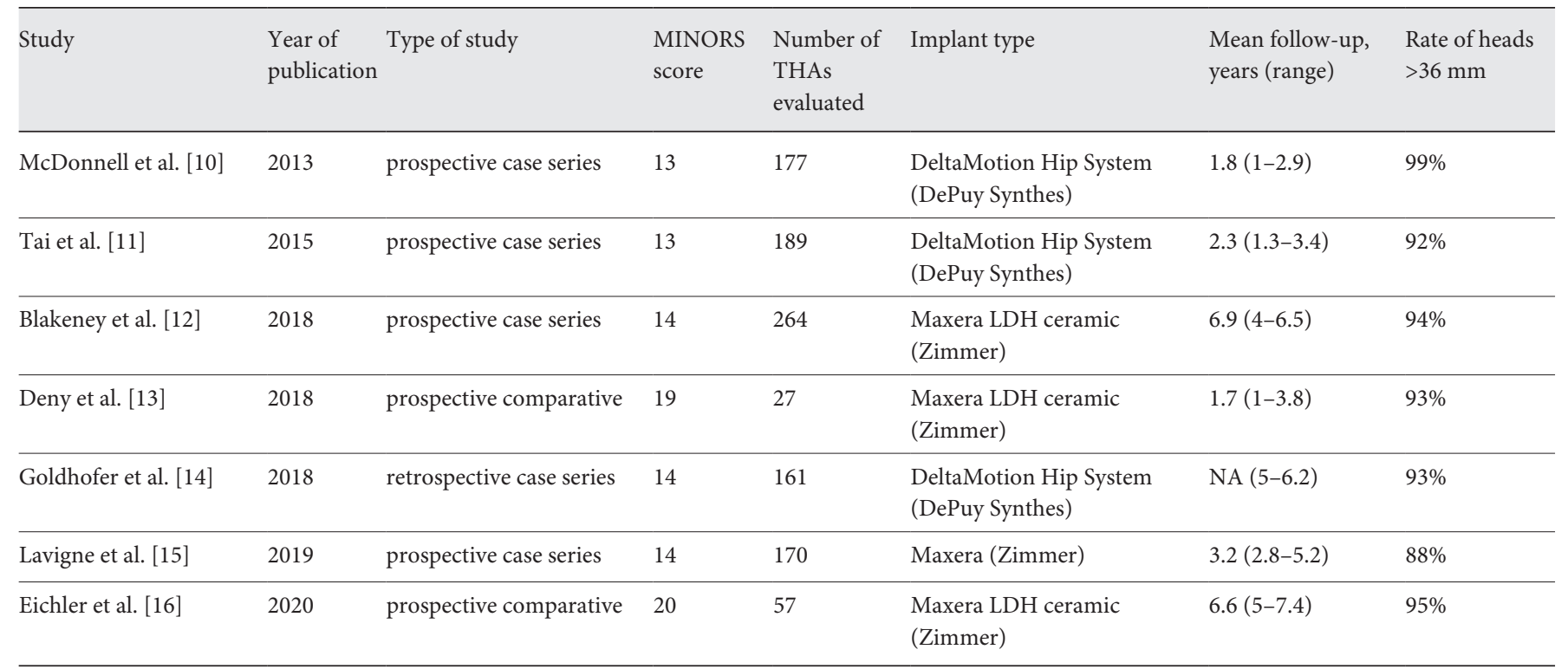

Scale values were generally positive, with no significant differences, apart from the symptoms and sport subsections of the HOOS. Deny et al. [13] provided a comparison between THAs with and without sleeves; no significant differences were detected in terms of clinical outcomes (Table 3). The mean UCLA Activity Scale score was 33.8 points and the mean WOMAC score was 8.8. Eichler et al. [16] updated and enlarged the same case series, reporting that no clinical differences could be appreciated at a minimum follow-up of 5 years.

\section{Groin Pain}

Goldhofer et al. [14] reported that $93 \%$ of the patients did not complain about groin or thigh pain (Table 3). Only Lavigne et al. [15] provided an adequate evaluation of groin pain (7.1\% of the patients reported moderate or mild pain). One patient $(0.6 \%)$ required an arthroscopic release of the psoas tendon. Groin pain significantly affected clinical outcomes and was prevalent in the female population.

\section{Noise}

Noise ranged from $3.5 \%$ to $36 \%$, but this large variability was frequently due to the research methodology ( $\mathrm{Ta}-$ ble 3) [10-16]. Blakeney et al. [12] described self-reported noise for 11 hips (4\%); this rose to $30 \%$ of the cases when a specific questionnaire was administered. Using a specific format to detect noise (the MONA questionnaire), McDonnell et al. [10] identified 65 noisy hips (31\%) at a mean follow-up of 21 months. Eichler et al. [16] reported a very high rate of noise (36\%) without administering specific questionnaires. All the papers reported that most of the noisy hips squeaked, with clicking accounting for the rest [10-16]. Noise was usually perceived after deep flexion movements [10-16]. However, McDonnell et al. [10] noticed that in $40 \%$ of cases, noise was not elicited by a specific movement. All the patients were satisfied with their THA in spite of noise.

The profile of the squeaking hip and correlations with demographics and implant-related features were debated further [10-16]. McDonnell et al. [10] stated that squeaking occurred in females with ligament laxity as well as in patients with a greater range of movement, smaller heads, or highly inclined cups with low anteversion (all of these findings were statistically significant).

In contrast, Tai et al. [11] and Goldhofer et al. [14] reported that squeaking tended to occur in larger heads (a mean head size of $44 \mathrm{~mm}$ ), with 48 -mm heads being noisy in $11.1 \%$ of the cases. Component positioning did not influence noise, even if the noisy hip tended to be outside the safe zone. Blakeney et al. [12] reported that squeaking occurred in younger and active male patients, with a larger contact patch-to-rim distance and a larger head size.

\section{Revision Rate}

Revision rates were very low; 4 papers out of 7 reported no revision at all (Table 3$)[10,13,15,16]$. A total of
Castagnini/Cosentino/Bracci/Masetti/ Faldini/Traina 
10 revisions were reported in the case series of Tai et al. [11] (included in Goldhofer et al. [14]). Two were early revisions due to acetabular migration and incorrect component positioning $[12,15]$. Five were due to periprosthetic fractures $[12,14]$. The remaining 3 were due to an acute periprosthetic joint infection, sciatic neuropathy, and traumatic dislocation $[12,14]$.

\section{Dislocations}

A very low rate of dislocations was reported. McDonnell et al. [10], Tai et al. [11], Goldhofer et al. [14], Lavigne et al. [15], and Eichler et al. [16] described only 1 case per case series, and they all occurred in neurological patients (with spina bifida or alcohol-related cerebellar ataxia), or after trauma. The case reported by Tai et al. [11] (and Goldhofer et al. [14]) required a surgical revision, with soft-tissue reconstruction and head-lengthening. Conservative treatment was performed in the other cases, with successful outcomes (Table 3 ) $[10,16]$.

\section{Aseptic Loosening}

No implant was revised due to aseptic loosening (Table 3) [10-16]. Tai et al. [11] reported that $1.5 \%$ of the cups showed stable fibrous growth and $1 \%$ of the stems showed minor radiolucency. Two stems (1\%) subsided. Similarly, Goldhofer et al. [14] noticed $0.9 \%$ radiolucency around the cup and $1.8 \%$ around the stem.

\section{Trunnionitis}

Deny et al. [13] evaluated the blood titanium and chromium-cobalt levels in 2 groups of unilateral THAs, with and without sleeves. The 2 groups were not homogenous for demographics. The THAs with sleeve interposition had a significantly lower values of blood ions, but neither group showed any clinical signs of trunnionitis. When the case series was enlarged and extended to a minimum follow-up of 5 years, the blood titanium level tended to decrease over time, and no differences could be detected between THAs with or without sleeve interposition [16]. Clinical or radiographic signs of trunnionitis were not reported (Table 3) [13, 16].

\section{Discussion}

The clinical outcomes of COC THAs with large heads were excellent in the vast majority of patients (Table 3) [10-16]. Large heads should theoretically match the native anatomy as closely as possible, i.e., $48 \mathrm{~mm}$ for females and $55 \mathrm{~mm}$ for males [4]. Although Zagra et al. [17] failed

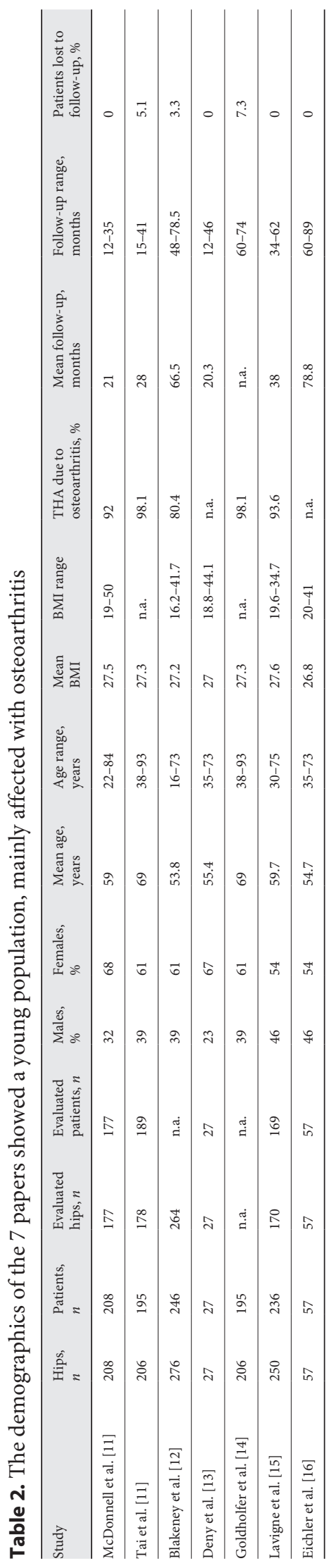


Table 3. Summary of the outcomes in the 7 papers (noise was the main complication by far)

\begin{tabular}{|c|c|c|c|c|c|c|c|c|}
\hline Study & Clinical outcome & $\begin{array}{l}\text { Excellent } \\
\text { results, \% }\end{array}$ & $\begin{array}{l}\text { Groin } \\
\text { pain, \% }\end{array}$ & $\begin{array}{l}\text { Noise, } \\
\%\end{array}$ & $\begin{array}{l}\text { Revision } \\
\text { rate, \% }\end{array}$ & $\begin{array}{l}\text { Dislocation, } \\
\%\end{array}$ & $\begin{array}{l}\text { Aseptic } \\
\text { loosening, \% }\end{array}$ & $\begin{array}{l}\text { Trunnionitis, } \\
\%\end{array}$ \\
\hline McDonnell et al. [10] & n.a. & n.a. & n.a. & 31 & 0 & 0.6 & 0 & n.a. \\
\hline Blakeney et al. [12] & 7.7 (WOMAC) & 99 & n.a. & 30 & 1.4 & 0 & 0 & n.a. \\
\hline Deny et al. [13] & 33.8 (UCLA) and 8.8 (WOMAC) & n.a. & n.a. & 7.4 & 0 & 0 & 0 & 0 \\
\hline Lavigne et al. [15] & 96.8 (HHS) & 98.8 & 7.1 & 3.5 & 0.6 & 0.6 & 0 & n.a. \\
\hline Eichler et al. [16] & 34.3 (UCLA) and 8.4 (WOMAC) & 98 & n.a. & 36 & 0 & 1.8 & 0 & 0 \\
\hline
\end{tabular}

Clinical outcomes are presented as scores of the assessment measures used, which appear in parentheses. HHS, Harris Hip Score; WOMAC, Western Ontario and McMaster Universities Osteoarthritis Index; UCLA, University of California LA Activity Scale; n.a., not available.

to demonstrate that large heads provide an improvement in gait in comparison to conventional heads in the same implant, in some of the papers involved in the review, many patients described the replaced hip as "natural," almost all patients were satisfied with the surgery, and more than half were very active and took part in sports (Table 3 ). Although the clinical findings were promising, the different outcome measures, the low number of patients involved, and the lack of comparisons did not allow us to draw any conclusions about large heads and "native hip" perception.

The most notable clinical concern about ceramic large heads is the risk of soft-tissue impingement and consequent groin pain, mainly due to the mechanical irritation of the iliopsoas tendon/capsule [18]. Only Lavigne et al. [15] reported a discrete rate of mild and moderate groin pain $(7.1 \%)$, which significantly impacted the clinical outcomes. Females were more involved. The rate of groin pain was probably underrated/inadequately investigated in most of the papers reviewed.

The papers providing the most accurate investigations about noise stated that up to $30 \%$ of the patients reported noise, squeaking in particular (Table 3). Noise was more frequently associated with extreme cup positioning, larger heads, younger and more active patients, and larger articular excursions (inconstant findings) [10-16]. The authors advocated many causative factors, among them edge-loading, dry conditions, a lower natural resonant frequency of the head-stem construct, and an increasing spine stiffness over the time [10-16]. Bishop et al. [6] demonstrated that large heads in COC couplings achieve high-friction moments in poor-lubrication conditions (5-fold); drier conditions may occur in cases of malposi- tioning. Instances of high friction may predispose to squeaking and minimum surface damage [5]. Thus, the high rate of noise in this paper might have been due to poor lubrication caused by many different factors (edge loading or malpositioning), but it was a complication of large-head COC implants. While noise was not found to influence the clinical outcomes and seemed to not cause any bearing surface failure, the rate of squeaking is not completely reassuring, as some sort of damage to the bearing surfaces may indeed ensue in the mid- to longterm $[5,9]$.

Dislocation is still one of the major complications occurring after THA; it accounts for $1.9 \%$ after 1 year $[4,19]$. At least half of the cases occur in the first year after surgery $[19,20]$. Large heads are believed to reduce the risk of dislocation and subluxation by increasing the jump distance and the head-to-neck ratio, resulting in a greater range of motion [2]. In a finite-element analysis, Kluess et al. [21] demonstrated that larger heads reduce the contact pressures at the egress site and increase the impingement-free range of motion. These preclinical findings were confirmed by Ertaş et al. [20], who evaluated the clinical benefit of a transition from 28-mm balls to larger heads (cross-linked polyethylene liners). Larger heads $(\geq 36 \mathrm{~mm})$ resulted in a much lower rate of dislocations (1.1 vs. $3.4 \%$ ), despite component positioning [20]. All the papers in this review reported a dislocation rate $<1 \%$ at short- to mid-term follow-ups (apart from the small case series by Eichler et al. [16]), and all of the dislocations occurred in neurological patients or after trauma [10-16]. Thus, large heads seemed to protect against dislocation, although the limited number of patients is not sufficient to provide any definitive evidence (Table 3) [10-16]. 
Aseptic loosening is another possible complication occurring with large heads due to high-friction moments that compromise bone anchorage $[2,5,22]$. Our review seems to be reassuring about this complication, with minimal and inconstant rates of aseptic loosening being reported (Table 3) [10-16].

Tapers are also a cause of concern with large-diameter heads. Mechanically assisted crevice corrosion is quite common with large-diameter heads in metal-on-metal THAs [4]. Titanium taper sleeves have been adopted to minimize the stresses on the trunnion with big heads. Deny et al. [13] and Eichler et al. [16] compared largehead-diameter THAs with and without sleeves, with the aim of detecting any differences concerning wear and corrosion. Implants with no sleeve interposed showed a significantly higher titanium ion release, decreasing over the time, with no clinical and radiological signs of trunnionitis $[13,16]$. Cobalt and chromium ions achieved minimal levels, and no signs of adverse local tissue reaction were detected [13]. This review did not reveal any signs of taperosis or adverse local tissue reactions (Table 3 ). Thus, no additional risk of taperosis seems to be evident with large head sizes in COC bearings, even in the mid-term.

To our knowledge, this is the first review dealing with large-head COC implants for THAs. The review has some notable limitations, related to the low number of cases, short follow-ups, and poor quality of the included articles (only 2 comparative studies). Moreover, the non-uniform data collection did not allow us to perform a meta-analysis. Most of the study limitations can be attributed to the quite recent introduction of these implants. However, this review, focusing on many aspects of large-head COC implants, does provide a comprehensive analysis of the outcomes, also giving hints for the design of future comparative clinical studies. Above all, we deem it important to focus on the precise clinical and radiological investigations of noise and groin pain. In addition, future biomechanical studies should include more investigation into lubrication films and component positioning.

\section{Conclusion}

This review supports the use of large-head COC THAs by confirming encouraging clinical outcomes, minimal revision rates, negligible dislocation rates, and no bearing surface damages at short- and mid-term follow-ups. However, the high rate of noise in this cohort (of up to $30 \%$ ) was not reassuring and deserves more investigation and long-term observation, possibly in the form of specifically designed, prospective studies.

\section{Disclosure Statement}

The authors have no conflicts of interest to declare.

\section{Author Contributions}

M.C., G.B., and C.M. collected the papers and analyzed the data. F.C., G.B., C.F., and F.T. designed the research and wrote the manuscript. F.T. and C.F. corrected and critically reviewed the manuscript.

\section{References}

1 Lombardi AV Jr, Skeels MD, Berend KR, Adams JB, Franchi OJ. Do large heads enhance stability and restore native anatomy in primary total hip arthroplasty? Clin Orthop Relat Res. 2011 Jun;469(6):1547-53.

2 Triclot P, Gouin F. Update. "Big-head": the solution to the problem of hip implant dislocation? Orthop Traumatol Surg Res. 2011 Jun;97(4 Suppl):S42-8.

3 Rodriguez JA, Rathod PA. Large diameter heads: is bigger always better? J Bone Joint Surg Br. 2012 Nov;94(11 Suppl A):52-4.

4 Tsikandylakis G, Mohaddes M, Cnudde P, Eskelinen A, Kärrholm J, Rolfson O. Head size in primary total hip arthroplasty. EFORT Open Rev. 2018 May;3(5):225-31.

5 [Internet] 2019 Australian registry report [cited 24 Nov 2019]. Available from: https://aoanjrr.sahmri.com/documents/10180/668596/Hi p $\% 2 \mathrm{C}+$ Knee+\%26+Shoulder+Arthroplasty/ c287d2a3-22df-a3bb-37a2-91e6c00bfcf0.

6 Bishop NE, Hothan A, Morlock MM. High friction moments in large hard-on-hard hip replacement bearings in conditions of poor lubrication. J Orthop Res. 2013 May;31(5): 807-13.

7 Moher D, Liberati A, Tetzlaff J, Altman DG; PRISMA Group. Preferred reporting items for systematic reviews and meta-analyses: the PRISMA statement. PLoS Med. 2009 Jul; 6(7):e1000097.

8 Slim K, Nini E, Forestier D, Kwiatkowski F, Panis Y, Chipponi J. Methodological index for non-randomized studies (minors): development and validation of a new instrument. ANZ J Surg. 2003 Sep;73(9):712-6.

9 Traina F, De Fine M, Di Martino A, Faldini C. Fracture of ceramic bearing surfaces following total hip replacement: a systematic review. BioMed Res Int. 2013;2013: 157247.

10 McDonnell SM, Boyce G, Baré J, Young D, Shimmin AJ. The incidence of noise generation arising from the large-diameter DeltaMotion ceramic total hip bearing. Bone Joint J. 2013 Feb;95-B(2):160-5.

11 Tai SM, Munir S, Walter WL, Pearce SJ, Walter WK, Zicat BA. Squeaking in large diameter ceramic-on-ceramic bearings in total hip arthroplasty. J Arthroplasty. 2015 Feb;30(2): 282-5.

12 Blakeney WG, Beaulieu Y, Puliero B, Lavigne M, Roy A, Massé V, et al. Excellent results of large-diameter ceramic-on-ceramic bearings in total hip arthroplasty: Is Squeaking Related to Head Size. Bone Joint J. 2018 Nov;100B(11):1434-41. 
13 Deny A, Barry J, Hutt JR, Lavigne M, Massé V, Vendittoli PA. Effect of sleeved ceramic femoral heads on titanium ion release. Hip Int. 2018 Mar;28(2):139-44.

14 Goldhofer MI, Munir S, Levy YD, Walter WK, Zicat B, Walter WL. Increase in benign squeaking rate at five-year follow-up: results of a large diameter ceramic-on-ceramic bearing in total hip arthroplasty. J Arthroplasty. 2018 Apr;33(4):1210-4.

15 Lavigne M, Vendittoli PA, Virolainen P, Corten K, Martinez M, Zicat B, et al. Large head ceramic-on-ceramic bearing in primary total hip arthroplasty: average 3-year follow-up of a multicentre study. Hip Int. 2019. DOI: 10.1177/1120700019863376.
16 Eichler D, Barry J, Lavigne M, Massé V, Vendittoli PA. No radiological and biological sign of trunnionosis with large diameter head ceramic bearing total hip arthroplasty after 5 years. Orthop Traumatol Surg Res. 2020. DOI: 10.1016/j.otsr.2019.12.015.

17 Zagra L, Anasetti F, Bianchi L, Licari V, Giacometti Ceroni R. No difference in gait recovery after THA with different head diameters: a prospective randomized study. Clin Orthop Relat Res. 2013 Dec;471(12):3830-7.

18 Tassinari E, Castagnini F, Mariotti F, Biondi F, Montalti M, Bordini B, et al. Arthroscopic tendon release for iliopsoas impingement after primary total hip arthroplasty: a retrospective, consecutive series. Hip Int. 2019. DOI: $10.1177 / 1120700019893341$.
19 Tamaki T, Oinuma K, Miura Y, Higashi H, Kaneyama R, Shiratsuchi H. Epidemiology of dislocation following direct anterior total hip arthroplasty: a minimum 5-year follow-up study. J Arthroplasty. 2016 Dec;31(12):2886-8.

20 Ertaş ES, Tokgözoğlu AM. Dislocation after total hip arthroplasty: does head size really matter? Hip Int. 2020. DOI: 10.1177/1120700019898404.

21 Kluess D, Martin H, Mittelmeier W, Schmitz $\mathrm{KP}$, Bader R. Influence of femoral head size on impingement, dislocation and stress distribution in total hip replacement. Med Eng Phys. 2007 May;29(4):465-71.

22 Nassutt R, Wimmer MA, Schneider E, Morlock MM. The influence of resting periods on friction in the artificial hip. Clin Orthop Relat Res. 2003 Feb;407:127-38. 